\title{
ANÁLISE DA CORRELAÇÃO DO LEAN MANUFACTURING COM PRODUTOS MODULARES
}

\section{THE ANALYSIS OF THE CORRELATION BETWEEN LEAN MANUFACTURING WITH MODULAR PRODUCTS}

Versão do autor aceita publicada online: $13 \mathrm{fev} .2020$

Publicado online: 12 maio 2021

Como citar esse artigo - American Psychological Association (APA):

Carnevalli, J. A., Georges, M. R. R., Benedicto, S. C., \& Sousa, J. E. R. (2021, abr./jun.). Análise da correlação do lean manufacturing com produtos modulares. Exacta, 19(2), 432-455. https://doi.org/10.5585/exactaep.2021.11316.

Submeta seu artigo para este periódico $\beta$

Dados Crossmark 


\section{ANÁLISE DA CORRELAÇÃO DO LEAN MANUFACTURING COM PRODUTOS MODULARES}

\section{THE ANALYSIS OF THE CORRELATION BETWEEN LEAN MANUFACTURING WITH MODULAR PRODUCTS}

\author{
${ }^{1}$ Pós doutorado em Engenharia de Produção \\ Pontifícia Universidade Católica de Campinas - PUC- \\ Campinas \\ jcarnevalli@hotmail.com \\ 2 Doutorado em Engenharia Mecânica \\ Pontifícia Universidade Católica de Campinas - PUC- \\ Campinas \\ marcos.georges@puc-campinas.edu.br \\ ${ }^{3}$ Doutorado em Administração \\ Pontifícia Universidade Católica de Campinas - PUC- \\ Campinas \\ samuel.benedicto@puc-campinas.edu.br \\ ${ }^{4}$ Doutorado em Administração \\ Pontifícia Universidade Católica de Campinas - PUC- \\ Campinas \\ jrsousa@gmail.com
}

Recebido em: 10 jan. 2019

Aprovado em: 13 fev. 2020
Resumo: A abordagem modular tem sido aplicada com bom desempenho na indústria automobilística e nos fabricantes de computadores que tradicionalmente utilizam o Lean Manufacturing. Mas qual é o seu efeito no Lean Manufacturing? Será que a modularidade está restrita somente a estes setores? Este trabalho responde a estas questões por meio de uma revisão da literatura e do uso dos diagramas de afinidade e de árvore em conjunto com a matriz de correlação. Nos resultados verificou-se o uso da modularidade na fabricação de máquinas industriais, residência, prédios, aviões, eletrodomésticos, brinquedos, bicicleta, software entre outros. Estes resultados mostram que a modularidade pode ser aplicada em vários tipos de produtos manufaturados. Em relação ao Lean Manufacturing a modularidade tem um efeito positivo nesta aplicação ajudando a atender alguns dos objetivos do Lean como redução do lead time.

Palavras-chave: Modularidade. Revisão de literatura. Lean Manufacturing.

Abstract: The modular approach has been applied with good performance in the automotive industry and in the computer manufacturers that traditionally use Lean Manufacturing. But what is its effect on Lean Manufacturing? Is modularity only restricted to these sectors? This paper answers these questions through a literature review and the use of the affinity diagram and the tree diagram together with the correlation matrix. In the results it was verified the use of modularity in the manufacturing of industrial machines, residences, buildings, airplanes, appliances, toys, bike, software among others. These results show that modularity can be applied to many types of manufactured products. Regarding Lean Manufacturing, the modularity has a positive effect on this application helping to meet some of the Lean's goals such as lead time reduction.

Keywords: Modularity. Literature review. Lean Manufacturing. 


\section{Introdução}

Um procedimento amplamente aplicado na indústria automobilística é a modularidade. Entretanto, tal abordagem tem sido utilizada não somente no setor automobilístico e de informática (fabricação de computadores), mas também em outros setores como a construção naval e a engenharia civil. Conforme verificado na literatura, no setor automobilístico tal abordagem ajuda a operacionalizar as estratégicas genéricas de liderança em custos e de diferenciação. Isto porque ao dividir os produtos em módulos é possível reduzir custos pelo uso de módulos padronizados em vários produtos ou conseguir a customização dos produtos pelo uso de módulos diferenciados. Além disso, a possibilidade de terceirizar o projeto, a fabricação e montagem de cada tipo de módulo para empresas diferentes vem alterando a estrutura da cadeia de suprimentos na busca de uma relação de maior parceria entre fornecedores e clientes e focando cada empresa nas suas competências essenciais. Mas qual é o efeito do uso da modularidade no Lean Manufacturing? A modularidade teria um efeito positivo ou neutro na aplicação de uma produção Lean Manufacturing? Será que a modularidade só é adequada para os setores automobilísticos, informáticos, naval e construção civil? Por esta razão, é importante realizar uma análise teórica da correlação da produção Lean Manufacturing com a modularidade e os setores que a aplicam, verificando a importância ou não deste procedimento junto com o Lean Manufacturing. Foram utilizados como método de análise o diagrama de afinidades, diagrama em árvore para organizar os dados em duas tabelas "benefícios da modularidade"; "elementos Lean Manufacturing" as quais foram utilizadas para formar uma matriz de correlação. O objetivo é verificar a correlação da modularidade com produção Lean Manufacturing; também é objetivo verificar se a estratégia contribui na aplicação do Lean Manufacturing. Será ainda verificado se o uso de módulos também é adequado para setores diferentes do automotivo e da indústria de computadores. As seguintes hipóteses foram analisadas:

Hipótese 1 do estudo: O uso da modularidade facilita a aplicação do Lean Manufacturing. Ou seja, apresenta correlação positiva.

Hipótese 2 do estudo: 0 uso da modularidade teria um efeito neutro na aplicação do Lean Manufacturing. Ou seja, não ajuda nem prejudica a aplicação.

\section{Método de pesquisa}

Este trabalho utilizou os dados de pesquisas bibliográficas organizados pelo método diagrama de afinidades, diagrama em árvore e analisados pela matriz de correlação, para verificar o efeito que a modularidade tem na produção Lean Manufacturing. 


\subsection{Pesquisa bibliográfica}

Foram realizadas as seguintes pesquisas bibliográficas com o uso de livros didáticos e artigos científicos:

- O método Lean Manufacturing;

- Adaptações na aplicação do Lean Manufacturing no Ocidente;

- Verificação de quais outros setores, além do automobilístico e de computadores, tem utilizado a estratégia modular;

- Revisão dos fichamentos sobre a abordagem modular realizados nas pesquisas passadas para identificar os conceitos, metodologias, etapas da aplicação que serão correlacionados com os conceitos, metodologias, etapas da aplicação do Lean Manufacturing.

Para a pesquisa bibliográfica foram utilizadas as seguintes etapas (Marconi, \& Lakatos, 2007): identificação, localização, obtenção, e cadastramento das fontes bibliográficas.

\subsection{Organização dos dados}

Os dados dos fichamentos sobre modularidade e os elementos do Lean Manufacturing foram estruturados separadamente em duas tabelas para formar a matriz de correlação. Para organizar estas duas tabelas utilizou-se os diagramas de afinidades e diagrama em árvore (Carnevalli, Salerno, \& Miguel, 2010; p. 8) com o seguinte procedimento:

a) Realizou-se, durante os fichamentos dos artigos estudados, a separação dos itens de interesse em planilhas do Excel $^{\circ}$ nos seguintes assuntos: [...] sendo os diagramas aplicados em cada um destes itens separadamente em tabelas.

b) Montou-se a tabela no Excel ${ }^{\circ}$, organizando os itens em grupos por ideias similares e por nível de abstração. Nos casos em que existiam itens repetidos, estes foram unidos em um só, mas mantendo as referências de todos os autores que os citaram;

c) Acrescentou-se, para cada grupo, um título que representasse a ideia do grupo, sendo que este título tinha um nível mais abstrato que o grupo que ele representava. Em vários casos, não foi necessário criar este título, pois já existia um item mais abstrato que os demais, o qual foi usado como título do grupo;

d) Agruparam-se também por ideias similares os títulos dos grupos definidos na etapa " $c$ ";

e) Repetiram-se as etapas "c $c$ " " $d$ " até organizarem todos os itens analisados;

f) Verificou-se se os níveis hierarquizados definidos nos agrupamentos estavam consistentes e foram feitas as correções, quando necessário;

g) Revisou-se e atualizou-se cada tabela com a análise de novos dados da literatura, respeitando os agrupamentos já realizados.

\subsection{Matriz de correlação}

Segundo Carvalho et al. (2012), as matrizes são utilizadas para organizar uma grande quantidade de dados para ser verificada a existência de correlação entre eles. 
Nesta pesquisa foi trabalhada uma matriz para verificar as correlações entre os dados das duas tabelas, sendo formada pelos benefícios da modularidade com os elementos do Lean Manufacturing em suas dimensões;

As correlações foram realizadas utilizando os seguintes valores: Facilita a aplicação da estratégia (forte: 9; médio: 3; fraca: 1) Ohfuji, Ono, \& Akao (1997); tem efeito neutro na aplicação da estratégia (0).

\section{Resultados}

Nesta seção serão apresentados os resultados da pesquisa.

\subsection{Elementos, metodologias e etapas da aplicação do Lean Manufacturing}

Após a análise da literatura, os dados foram organizados utilizando os diagramas de afinidades e diagrama em árvore. Os principais resultados estão na tabela 1, onde foram identificados os elementos, metodologias e as etapas da aplicação do Lean Manufacturing, os quais estão relacionados com:

1. Flexibilidade (Barbosa, Carvalho, \& Filho, 2014, p. 1260). De modo a permitir alterar rapidamente o mix de produção conforme a demanda real e trabalhar com pequenos lotes, o que é possível com a redução do tempo de set up;

2. Função do operário no Lean Manufacturing (Womack, Jones, \& Roos, 1992). Diferente da produção em massa, o Lean Manufacturing incentiva o trabalho em equipes e a possibilidade do operário realizar atividades diferentes na linha de produção por ser multiqualificado;

3. Parceria entre montadora com seus fornecedores (Arnheiter, \& Greenland, 2008; Womack, et al., 1992). O Lean Manufacturing já na década de 1960 incentivava a criação de parceria com seus fornecedores, o que já permitia naquela época terceirizar parte do desenvolvimento do produto para esses fornecedores.

4. Melhoria do layout (Chaplin, \& O'rourke, 2016). A busca do layout mais eficiente também ocorre no Lean Manufacturing, devido a busca de reduzir tempos não produtivos, como os tempos de movimentação das peças na linha de produção.

5. Análise do Fluxo de Valor (Carmignani, \& Zammori, 2015 p. 994; Lopes, Freitas, \& Sousa, 2015; Jasti, \& Sharma, 2014). O mapeamento do fluxo de valor para reduzir atividades não produtivas é um dos destaques da produção Lean Manufacturing;

a. Melhoria da qualidade (Chaplin, \& O'rourke, 2016). Gestão da qualidade (Cagatay, 2014). A gestão e melhoria da qualidade são pontos fundamentais para viabilizar a aplicação do Just in time que é uma 
parte do Lean Manufacturing. Isto porque a busca do estoque "zero" no Just in time depende fortemente de uma drástica redução no número de peças e produtos com defeitos, além de máquinas da linha de produção confiáveis, com baixa possibilidade de quebra e de se ter fornecedores confiáveis, que cumprem os prazos de entrega.

Após a análise da tabela 1 verifica-se que o uso de módulo já aparece como elemento do Lean Manufacturing no trabalho de Womack, et al. (1992), inclusive na terceirização do projeto e produção, para os seus fornecedores de primeiro nível. Mas estes módulos eram chamados de subconjuntos, podendo não ter as mesmas características detalhadas por Baldwin e Clark (2000), quando apresentaram os princípios do projeto modular desenvolvidos pela IBM na década de 1960. De qualquer modo, se verifica que o uso de fornecedores para projetar e entregar subconjuntos na linha de montagem não é uma estratégia recente, incorporada pela indústria automobilística nos anos 1990 com base nos exemplos da indústria de informática, mas sim uma prática antiga que vem do Japão desde a década de 1960.

Tabela 1 - Elementos da Produção Lean Manufacturing quando comparados com a produção em massa

(continua)

\section{Nível $1 \quad$ Nível 2}

\begin{tabular}{|c|c|c|}
\hline Nível 1 & Nível 2 & Nível 3 \\
\hline \multirow[t]{2}{*}{$\begin{array}{l}\text { Flexibilidade (Barbosa } \\
\text { et al., 2014, p. 1260). }\end{array}$} & $\begin{array}{l}\text { 个 Máquinas Flexíveis } \\
\text { (Womack, et al.,1992). }\end{array}$ & $\begin{array}{l}\text { (L1): Sistemas de troca rápida de ferramentas (Cagatay, 2014). } \\
\text { (L2): Flexibilidade: Assegurar processar diferentes produtos. } \\
\text { (Barbosa et al., 2014, p. 1260). } \\
\text { (L3): Flexibilidade: Uso de robôs industriais que possam fazer } \\
\text { produtos diferentes (Barbosa et al., 2014, p. 1260). } \\
\text { (L4): } \uparrow \text { Máquinas automatizadas (Womack, et al., 1992). }\end{array}$ \\
\hline & 5): $\uparrow$ Variedade de produ & dutos (Womack, et al., 1992) \\
\hline \multirow[t]{4}{*}{$\begin{array}{l}\text { Função do operário } \\
\text { na produção enxuta } \\
\text { (Womack, et al., } \\
\text { 1992). }\end{array}$} & $\begin{array}{l}\text { Trabalho em equipes } \\
\text { multifuncionais } \\
\text { (Womack, et al., 1992) }\end{array}$ & $\begin{array}{l}\text { (L6): Desenvolvimento de pessoas, para o trabalho em equipe } \\
\text { (Arnheiter, \& Greenland, 2008). } \\
\text { (L7): Melhoria da produtividade dos funcionários (Chaplin, \& } \\
\text { O'rourke, 2016). } \\
\text { (L8): Maior participação dos funcionários na solução de } \\
\text { problemas (Chaplin, \& O'rourke, 2016) } \\
\text { (L9): Maior participação dos funcionários nas sugestões de } \\
\text { melhorias (Chaplin, \& O'rourke, 2016). } \\
\text { (L10): } \text { 个 Qualidade (Womack, et al., 1992). } \\
\text { (L11): } \text { 个 Estimulante (Womack, et al., 1992). }\end{array}$ \\
\hline & \multirow{3}{*}{$\begin{array}{l}\text { 个Trabalhadores } \\
\text { Multiqualificados } \\
\text { (Womack, et al., 1992) }\end{array}$} & (L12): $\uparrow$ Estresse (Womack, et al., 1992). \\
\hline & & (L13): $\uparrow$ Responsabilidade (Womack, et al., 1992). \\
\hline & & $\begin{array}{l}\text { de dos trabalhadores, para realizar várias tarefas } \\
\text { 1992). }\end{array}$ \\
\hline $\begin{array}{l}\text { Parceria entre } \\
\text { montadora com seus } \\
\text { fornecedores } \\
\text { (Arnheiter, \& }\end{array}$ & $\begin{array}{l}\text { Fornecedores de } \\
\text { primeiro nível (Womack, } \\
\text { et al., 1992) }\end{array}$ & $\begin{array}{l}\text { (L15): Participação no Projeto do subconjunto (Womack, et al., } \\
\text { 1992). } \\
\text { (L16): O fornecedor faz o protótipo do produto (Womack, et al., } \\
\text { 1992). }\end{array}$ \\
\hline
\end{tabular}


Greenland, 2008;

Womack, et al.,

1992). (conclusão)

(L17): Fornecedores de diferentes componentes não concorrentes trocam o conhecimento (Womack, et al., 1992).

(L18): Recebendo especificações e o valor que deve custar. (Womack, et al., 1992).

(L19): Fornecedor de subconjunto (Womack, et al., 1992).

(L20): Proximidade das instalações físicas dos clientes (Womack, et al., 1992).

(L21): Fabricam as peças individuais (Womack, et al., 1992).

Fornecedores de segundo (L22): Cada um é especializado em peças diferentes, eles não são nível (Womack, et al., concorrentes (Womack, et al., 1992).

1992)

(L23): Contribuem uns com os outros na melhoria dos processos de fabricação (Womack, et al., 1992).

Layout para minimizar o uso do espaço são regras

já padrão de layout

Melhoria do layout

(Chaplin, \& O'rourke, 2016). (minimizar área utilizada devido ao seu alto custo e manter a flexibilidade da linha). (Barbosa et al., 2014, p. 1260).

(L24): Fluxo Contínuo (Gong, \& Janssen, 2015, p. 371; Barbosa et al., 2014, p. 1260).

(L25): Fluxo mais integrado (Barbosa et al., 2014).

(L26): Sistema mais confiável (Barbosa et al., 2014).

(L27): Sistema mais rápido (Barbosa et al., 2014).

(L28): Células de manufaturas (Cagatay, 2014).

(L29): O uso do SMED ajudou na redução do tempo improdutivo (Bevilacqua, Ciarapica, De

Análise do Fluxo de Sanctis, Mazzuto, \& Paciarotti, 2015; Lopes et al., 2015, p. 125).

valor (Carmignani, \& Zammori, $2015 \mathrm{p}$.

(L30): O mapeamento de valor e o kaizen melhoram o processo de fabricação, (Jasti, \& Sharma, 2014).

994); SMED (Lopes et al., 2015).

$\downarrow$ Estoques (Womack, et (L31): O mapeamento de valor e o kaizen reduziu os estoques, O mapeamento de al., 1992). (Jasti, \& Sharma, 2014). valor (Jasti, \& Sharma, 2014).

Eliminação dos desperdícios (Chaplin, \& O'rourke, 2016).

(L32): Identificação e eliminação de desperdícios (Carmignani, \& Zammori, 2015 p. 994).

Melhoria da qualidade (Chaplin, \& O'rourke, 2016).

Gestão da qualidade (Cagatay, 2014).

Produção puxada pela demanda (Womack, et al., 1992).

(L33): Produção puxada. Foco na qualidade (parar a produção para corrigir erros).

Produção puxada (Gong, \& Janssen, 2015, Cagatay, 2014; Zarei, \& Fakhrzad; Paghaleh, 2011).

Houve melhoria econômica (Chaplin, \& O'rourke, 2016).

Fonte: Elaborada pelos autores.

Deste modo, verifica-se que o Lean Manufacturing apresenta vários benéficos ao ser comparado com a produção em massa, tais como produções mais flexíveis, melhorias da qualidade e redução dos estoques, funcionários mais participativos e aumento da parceria com os fornecedores. 


\subsection{Adaptações na aplicação do Lean Manufacturing no Ocidente}

Em relação às adaptações na aplicação do Lean Manufacturing no Ocidente, foram identificadas algumas adaptações nos seguintes estudos:

No setor aeroespacial, Barbosa et al. (2014) desenvolveram uma estrutura guia para projeto de processos automatizados de fabricação, utilizando os requisitos e princípios da filosofia Lean Manufacturing (Barbosa et al., 2014). Foram considerados equipamentos, operários, layout, produtividade, flexibilidade e segurança num projeto de automação. Neste caso, verifica-se um esforço de se adaptar o Lean Manufacturing para um setor onde a escala de produção é bem menor quando comparada com a indústria de informática e automobilística. Entretanto, o sucesso desta aplicação era esperado, visto que é um setor que trabalha num ambiente de fabricação sob encomenda (MTO: make to order) próprio para o uso do Lean Manufacturing.

A aplicação do Lean Manufacturing na indústria automobilística, instalada na África do Sul, foi realizada com a necessidade de adaptações. Segundo os pesquisadores Morgan e Liker (2006 como citado em Mund, Pieterse, \& Cameron, 2015), na África do Sul os seguintes itens foram adaptados:

- Uso da Engenharia Simultânea, para explorar soluções alternativas de projetos e maximizar espaço no projeto;

- Realização de vários treinamentos, para desenvolver um chefe de engenharia líder que acompanha o projeto integrado do começo ao fim;

- Adequação do processo seletivo e do treinamento, para desenvolver altas competências técnicas em todos os engenheiros;

- Adaptação da tecnologia, e dos subsistemas de ferramentas às necessidades das pessoas e dos processos;

- Alinhamento da organização através de comunicação simples e visual;

- Padronização e aprendizagem organizacional na seleção e integração de ferramentas e tecnologia adaptadas às necessidades dos processos.

No trabalho de Morgan e Liker (2006 como citado em Mund et al., 2015), fica claro a importância do desenvolvimento do líder de equipe de projeto e o aumento do nível de treinamento dos engenheiros, para aplicar o Lean Manufacturing.

No trabalho de Carmignani e Zammori (2015) foi realizada uma adaptação do Lean Manufacturing para ser aplicado no mercado de luxo de confecção e vestuário. Para esta implantação, os autores, criaram a seguinte estrutura adaptada do Lean Manufacturing (Carmignani, \& Zammori, 2015 p. 994): 
1. Planejamento de projeto:

- Formação de equipes (uso da Matriz de habilidade de trabalhadores);

- Agrupamento dos objetivos (uso do SMART - Simple multi-attribute rating technique);

- Agendamentos (uso do gráfico de Gantt; Matriz de Responsabilidade; Matriz de Estrutura de projeto; Estrutura analítica de Projeto).

2. Análise do Fluxo de valor:

- Seleção do Fluxo de valor (usa técnicas de formação de famílias e Pareto);

- Observação direta;

- Mapeamento do estado atual e identificação de desperdícios aplicando Mapeamento do Fluxo de Valor e diagrama;

3. Identificação e eliminação de desperdícios:

- Análise de Causa e Efeito com a aplicação do FMEA, Ishikawa e Estratificação de Problemas;

- Elaborações de ações corretivas aplicando o 5S; Administração Visual; grupo focal e "lateral thinking";

- Priorizações de ações corretivas com a Matriz de Análise de Riscos;

- Mapeamento do estado Futuro aplicando o Mapeamento do Fluxo de valor;

4. Implementação e consolidação da implantação através de um projeto piloto e da definição de indicadores de performance chave, para avaliar os resultados.

No caso do mercado de luxo de confecção e vestuário, as adaptações estão em criar um roteiro de aplicação com a definição em sequência, dos objetivos de algumas das ferramentas da qualidade e do Lean Manufacturing, que devem ser aplicadas apesar de algumas delas não serem conhecidas no setor.

Forno et al. (2016) desenvolveram procedimentos para avaliar o Lean Manufacturing no desenvolvimento de produto e ajudar a empresa a identificar os pontos fracos e fortes, para melhorar a implantação. Para isto, os autores combinaram o método Benchmarking com o Lean.

Num estudo de caso, os autores verificaram os benefícios e risco do uso do Lean Manufacturing em serviços de inovação de um banco (Gong, \& Janssen, 2015). Neste caso, os conceitos de Kanban e 
de sistema puxado foram utilizados no desenvolvimento de produtos, como serviços on line, o que envolve desenvolver produtos dentro do digital banking, celular com aplicativos APP e web site. Ou seja, a inovação neste setor está em entregar serviços de atendimento on line para o cliente. O Lean de inovação envolve os seguintes princípios adaptados: "criar valor para o cliente"; pensamento sistemático; fluxo puxado e continuo, com a redução de interrupções, retrabalho e melhoria continua (Gong, \& Janssen, 2015, p. 371). Segundo os autores, estes elementos facilitam o conhecimento acessível e integrado, o que ajuda a criar serviços inovadores. Entretanto, verificou-se a necessidade de ter especialistas dentro das equipes, provavelmente por não conseguirem montar uma equipe multifuncional, como ocorre no setor industrial. Outro problema é a busca pelo excesso de padronização, o que pode limitar a criatividade (Gong, \& Janssen, 2015). Essas adaptações e limitações da equipe prejudicaram a aplicação.

\subsection{Outros setores que tem utilizado a modularidade}

Foram revisados os fichamentos sobre modularidade, realizados nas pesquisas passadas e com os novos fichamentos foi possível verificar quais são os outros setores que tem utilizado a modularidade e se nesses setores predomina a produção em massa ou a Lean Manufacturing. Para identificar a abordagem de produção predominante utilizou-se a seguinte classificação de Martins e Laugeni (2010): MTS: make to stock; MTO: make to order; ATO: assemble to order; ETO: engineering to order, sendo que a primeira se relaciona com a produção em massa e as demais tem forte correlação com o Lean Manufacturing.

A tabela 2 apresenta estes resultados. Deste modo, verifica-se a alta aplicabilidade da modularidade em diferentes setores e ambientes de produção sendo que, na maioria dos casos, predominou ambientes de manufatura com forte correlação com o Lean Manufacturing. O Lean Manufacturing junto com a modularidade se destacou nos setores de robótica, máquinas CNC, tratores, aviões, softwares, construção civil, estações de trabalho, micro eletromecânico e prótese biônica. No caso de fabricação de bicicleta na China, fica claro no artigo de Brookfield, Liu, \& MacDuffie, (2008) que se trata de produção com Lean Manufacturing. Entretanto, no setor de fabricação de bicicleta tradicionalmente este setor se caracteriza no ambiente de Manufatura de Fabricação para estoque (MTS: make to stock), ou seja, cadeia de suprimento empurrada e produção em massa. 
Tabela 2 - Diferentes setores de aplicação da Modularidade

\begin{tabular}{|c|c|c|}
\hline Setor de aplicação & Autores & $\begin{array}{l}\text { Estratégia de produção } \\
\text { predominante }\end{array}$ \\
\hline $\begin{array}{c}\text { Máquinas industriais CNC e setor de } \\
\text { robótica }\end{array}$ & $\begin{array}{l}\text { (Connolly, 2007; Ranky, 2007; } \\
\text { Moeckel et al., 2006; Virk, Muscato, } \\
\text { Semerano, Armanda, \& Warren, } \\
\text { 2004) }\end{array}$ & \multirow[t]{2}{*}{ Lean Manufacturing } \\
\hline Tratores tipos Escavadeira & $\begin{array}{c}\text { (Nepal, Monplaisir, \& Famuyiwa, } \\
\text { 2012) }\end{array}$ & \\
\hline $\begin{array}{l}\text { Aeronáutico (na fabricação de } \\
\text { aviões) }\end{array}$ & (Barbosa et al., 2014) & \multirow{2}{*}{$\begin{array}{c}\text { (MTO: make to order)* tem } \\
\text { forte correlação com o Lean } \\
\text { Manufacturing }\end{array}$} \\
\hline Prótese biônica (Prótese da mão) & (Connolly, 2008) & \\
\hline $\begin{array}{l}\text { Eletrodomésticos (produtos da linha } \\
\text { branca) }\end{array}$ & (Cagatay, 2014) & \multirow{2}{*}{$\begin{array}{l}\text { (MTS: make to stock)* não } \\
\text { utilizando o Lean } \\
\text { Manufacturing }\end{array}$} \\
\hline Produtos de plásticos e brinquedos & (Lau, Yam, \& Tang, 2007) & \\
\hline $\begin{array}{l}\text { Sistemas micro eletromecânico } \\
\text { (sistemas de controle inteligente de } \\
\text { pressão modular) }\end{array}$ & (Shuenemann et al., 1999) & \multirow[t]{2}{*}{$\begin{array}{c}\text { Não foi possível identificar o } \\
\text { ambiente de Manufatura } \\
\text { utilizada }\end{array}$} \\
\hline $\begin{array}{l}\text { Projeto de estações de trabalho } \\
\text { modulares, para linha de montagem }\end{array}$ & (Spath, \& Baumeister, 2001) & \\
\hline $\begin{array}{l}\text { Construção civil (projeto de } \\
\text { estacionamento em módulos } \\
\text { metálicos; Construção de casas) }\end{array}$ & $\begin{array}{c}\text { (Rezende, \& Gouveira, 2006; } \\
\text { Voordijk, Meijboom, \& Haan, 2006) }\end{array}$ & \multirow[t]{2}{*}{$\begin{array}{c}\text { (ETO: engineering to order)* } \\
\text { tem forte correlação com o } \\
\text { Lean Manufacturing }\end{array}$} \\
\hline Desenvolvimento de software & $\begin{array}{l}\text { (Gabrieli, Cortimiglia, \& Ribeiro, } \\
\text { 2007; Fong, \& Hui, 2006; Lohse, } \\
\text { Ratchev, \& Valtchanov, 2004) }\end{array}$ & \\
\hline Fabricação de bicicleta & (Brookfield et al., 2008) & $\begin{array}{l}\text { No artigo fica evidente o uso } \\
\text { do Lean Manufacturing com } \\
\text { ambiente de Manufatura ATO } \\
\text { (assemble to order)* }\end{array}$ \\
\hline
\end{tabular}

*Considerando a classificação de Martins e Laugeni (2010).

Fonte: Elaborada pelos autores a partir das fontes citadas.

Os resultados da Tabela 2 indicam a flexibilidade do uso da modularidade não somente em relação aos tipos de produtos, mas também em relação às abordagens de produção MTS: make to stock; MTO: make to order; ATO: assemble to order; ETO: engineering to order. Deste modo, verifica-se o uso da modularidade em todas as abordagens de produção, o que não significa que ela seja a mais indicada para qualquer abordagem. Um futuro estudo poderia verificar os efeitos da modularidade MTS: make to stock, que tem uma abordagem diferente do Lean Manufacturing.

A seguir são apresentados os resultados da aplicação da matriz de correlação. 


\subsection{Resultados da aplicação da matriz de correlação}

Dos 106 itens da modularidade, que foram identificados com a revisão dos fichamentos realizados nas pesquisas passadas e com os novos fichamentos, são apresentados os 30 itens que tiveram os maiores valores do total das correlações. Estes dados foram organizados usando os diagramas de afinidades e diagrama em árvore e formam as colunas e linhas da matriz, sendo as colunas os benefícios da modularidade e as linhas os 37 elementos Lean Manufacturing. Em relação às colunas verificam-se os seguintes elementos:

- (MA): Possibilitar a customização em massa (Machado, \& Moraes, 2008; Mondragon, Mondragon, \& Miller, 2006; Danese, \& Romano, 2004; Hoek, \& Weken, 1998). O uso de módulos com interfaces padronizadas permite alterar as características de um produto facilmente, com a troca de um único módulo;

- (MB): Aumentar a variedade de produtos (combinação de diferentes módulos num produto plataforma) (Acaccia, Bruzzone, \& Razzoli, 2008; Orsato, \& Wells, 2007; Ranky, 2007; Fredriksson, 2006a; Moeckel et al., 2006; Mondragon et al., 2006; Morris, \& Donnelly, 2006; Frigant, \& Lung, 2002; Yigit, Ulsoy, \& Allahverdi, 2002; Lee, Lau, \& Tam, 2001; Sanchez" , \& Collins, 2001; He, \& Kusiak, 1998; Hoek, \& Weken, 1998);

- (MC): Permite ter flexibilidade da linha de manufatura, para variação de demanda e de produto (Spath, \& Baumeister; 2001). Esta flexibilidade está relacionada ao mix de produtos, que podem ser facilmente alterados com a instalação de diferentes módulos.

- (MD): Possibilita a terceirização do desenvolvimento de alguns módulos (Fredriksson, 2006b). Com isso é possível reduzir o tempo de desenvolvimento de produto, por cada módulo ser realizado em empresas diferentes em paralelo;

- (ME): Possibilita a redução do tempo de reprojetar e do tempo de novos projetos, pelo aproveitamento de módulos já existentes (Moeckel et al., 2006; Lau, \& Yam, 2005; Zhang, Zhang, \& Xu, 2002; Moore, 1999);

- (MF): Redução de tempo de produção, pelos módulos poderem ser fabricados e montados de forma independente (Veloso, \& Fixson, 2001; Gu, \& Sosale 1999);

- (MG): Redução do lead time (O'gragy, \& Liang, 1998). Isto ocorre quando os módulos são fabricados e montados de forma independente;

- (MH): Apresentar correlação positiva com a porcentagem de entrega em just-in-time (Pérez, \& Sánchez, 2000);

- (MI): Reduzir o investimento na cadeia de suprimentos, pois é realizada pelos fornecedores de módulos (Doran, 2005); 
- (MJ): Reduz a linha de montagem da montadora, transferindo as submontagens dos módulos para os fornecedores (Connolly, 2007; Holmes, 2004). Sendo este um efeito da terceirização de atividades para o fornecedor;

- (MK): Reduz os estoques na linha de montagem, pela transferência da montagem dos módulos para os fornecedores (Doran, 2002);

- (ML): Participação dos fornecedores no projeto do produto com divisão de riscos (Brookfield et al., 2008). Isto porque o fornecedor investiu no projeto do módulo e só recebe o retorno deste investimento quando vende o produto para a montadora. Pois os custos de projetos dos módulos estão rateados nos preços de venda.

- (MM): Transfere em alguns casos a responsabilidade pelo desenvolvimento e o aperfeiçoamento dos módulos para os fornecedores (Holmes, 2004);

- (MN): Reduzir o número de peças a serem fabricadas em famílias de produto (Yigit et al., 2002);

- (MO): Módulos padronizados são utilizados em veículos diferentes (Interfaces padronizadas) (Morris, \& Donnelly, 2006). Isto reduz os custos da customização;

- (MP): Diminuir o número de subconjuntos diferentes (Moore, 1999);

- (MQ): Reduzir os custos de montagem, devido os fornecedores fazerem as submontagens dos módulos (Orsato, \& Wells, 2007; Fredriksson, 2006a; Mondragon et al., 2006);

- (MR): Reduzir custos pela padronização de módulos entre diferentes "marcas" (Batchelor, 2006). O que permite uma maior produção destes módulos comuns entre as marcas;

- (MS): Reduzir os custos de produção em produtos plataformas (Pandremenos, Paralikas, \& Chryssolouris, 2009);

- (MT): Reduzir os custos da customização em massa (Arnheiter, \& Harren, 2005). Devido a facilidade de alterar o produto mudando somente alguns módulos;

- (MU): Os fornecedores podem ajudar na redução de custos (por desenvolver os módulos) (Fredriksson, 2006b). Ao ficarem responsáveis pelos custos de projetos e fabricação dos módulos;

- (MV): O consórcio modular ou condomínio reduz os custos de serviços, como de resolução de problemas na linha de montagem da montadora (Salerno, Camargo, \& Lemos, 2008);

- (MW): Reduz de custos devido a melhoria da logística (De Mello, \& Mark, 2007);

- (MX): Reduz os custos de manutenção (Connolly, 2008; Cepolina, \& Michelini, 2003). Pois é mais fácil realizar a manutenção de um produto trocando os módulos defeituosos por novos;

- (MY): Simplificar a cadeia de suprimentos, por reduzir o número de fornecedores (Miguel, \& Pires, 2006; Arnheiter, \& Harren, 2005, 2006; Doran, 2002, 2005; Pires, 1998); 
- (MZ): Aumentar do valor do produto agregado (Collins, Bechler, \& Pires, 1997). Pois a empresa não fabrica só peças e sim produtos mais complexos e caros que são os módulos;

- (M1): Tem aumentado os lucros (fornecedor de $1^{\circ}$ nível) (Salerno et al., 2008);

- (M2): Participação dos fornecedores no projeto com divisão de lucros (Brookfield et al., 2008).

O que permite a criação de forte parceria entre as empresas;

- (M3): Exclusividade de fornecimento (proponente);

- (M4): Criar contratos de longos períodos com as montadoras, o que diminui a concorrência (Arnheiter, \& Harren, 2005; Pires, 2002; Salerno, 2001; Collins et al., 1997; Marx, Zilbovicius, \& Salerno, 1997) e consequentemente aumenta a parcerias entre as empresas.

A Figura 1 apresenta a matriz de correlação formada com os dados da tabela 1 e com os 8 itens de modularidade que apresentaram maior valor na correlação.

Figura 1 - Matriz de correlação com os itens mais importantes

\begin{tabular}{|c|c|c|c|c|c|c|c|c|}
\hline & & & & & & & & (continua) \\
\hline Lean Manufacturing/Modularidade & MA & $\mathrm{MC}$ & MG & $\mathrm{MH}$ & MT & MV & M3 & M4 \\
\hline $\begin{array}{l}\text { Sistemas de troca rápida de ferramentas } \\
\text { (Cagatay, 2014). }\end{array}$ & 3 & 3 & 9 & 1 & 9 & 0 & 0 & 0 \\
\hline $\begin{array}{l}\text { Flexibilidade: Assegurar processar diferentes } \\
\text { produtos (Barbosa et al., 2014, p. 1260). }\end{array}$ & 3 & 9 & 0 & 0 & 3 & 0 & 0 & 0 \\
\hline $\begin{array}{l}\text { Flexibilidade: Uso de robôs industriais, que } \\
\text { possam fazer produtos diferentes (Barbosa et } \\
\text { al., 2014, p. 1260). }\end{array}$ & 3 & 9 & 0 & 0 & 3 & 0 & 0 & 0 \\
\hline $\begin{array}{l}\text { 个 Máquinas automatizadas (Womack, et al., } \\
\text { 1992). }\end{array}$ & 3 & 9 & 0 & 0 & 3 & 0 & 0 & 0 \\
\hline $\begin{array}{l}\uparrow \text { Variedade de produtos (Womack, et al., } \\
1992 .\end{array}$ & 9 & 0 & 0 & 0 & 0 & 0 & 0 & 0 \\
\hline $\begin{array}{l}\text { Desenvolvimento de pessoas, para o trabalho } \\
\text { em equipe (Arnheiter, \& Greenland, 2008). }\end{array}$ & 0 & 0 & 1 & 0 & 0 & 0 & 0 & 0 \\
\hline $\begin{array}{l}\text { Melhoria da produtividade dos funcionários } \\
\text { (Chaplin, \& O'rourke, 2016). }\end{array}$ & 0 & 0 & 1 & 1 & 0 & 0 & 0 & 0 \\
\hline $\begin{array}{l}\text { Maior participação dos funcionários nas } \\
\text { soluções de problemas (Chaplin, \& O'rourke, } \\
\text { 2016). }\end{array}$ & 0 & 0 & 3 & 0 & 0 & 9 & 0 & 0 \\
\hline $\begin{array}{l}\text { Maior participação dos funcionários nas } \\
\text { sugestões de melhorias (Chaplin, \& O'rourke, } \\
\text { 201616). }\end{array}$ & 0 & 0 & 0 & 0 & 0 & 1 & 0 & 0 \\
\hline 个 Qualidade (Womack, et al., 1992). & 0 & 3 & 3 & 9 & 3 & 9 & 0 & 0 \\
\hline 个 Estimulante (Womack, et al., 1992). & 0 & 0 & 0 & 0 & 0 & 0 & 0 & 0 \\
\hline 个 Estresse (Womack, et al., 1992). & 0 & 3 & 0 & 0 & 0 & 0 & 9 & 0 \\
\hline 个 Responsabilidade (Womack, et al., 1992). & 0 & 1 & 0 & 3 & 0 & 0 & 9 & 9 \\
\hline $\begin{array}{l}\text { Flexibilidade dos trabalhadores, para realizar } \\
\text { várias tarefas (Womack, et al., 1992). }\end{array}$ & 3 & 3 & 3 & 3 & 3 & 0 & 0 & 0 \\
\hline
\end{tabular}


Participação no Projeto do subconjunto (Womack, et al., 1992).

O fornecedor faz o protótipo do produto (Womack, et al., 1992).

Fornecedores de diferentes componentes não concorrentes, trocam o conhecimento (Womack, et al., 1992).

Recebendo especificações e o valor que deve custar. (Womack, et al., 1992).

Fornecedor de subconjunto (Womack, et al., 1992).

Proximidade das instalações físicas dos clientes (Womack, et al., 1992).

Fabricam as peças individuais (Womack, et al., 1992).

Cada um é especializado em peças diferentes, eles não são concorrentes (Womack, et al., 1992).

Contribuem uns com os outros na melhoria dos processos de fabricação (Womack, et al., 1992).

Fluxo Contínuo (Gong, \& Janssen, 2015, p. 371; BARBOSA et al., 2014, p. 1260).

Fluxo mais integrado (Barbosa et al., 2014).

Sistema mais confiável (Barbosa et al., 2014).

Sistema mais rápido (Barbosa et al., 2014).

Células de manufaturas (Cagatay, 2014).

O uso do SMED ajudou na redução do tempo improdutivo (Bevilacqua et. al., 2015; Lopes et al., 2015, p. 125).

O mapeamento de valor e o kaizen melhoram o processo de fabricação, (Jasti, \& Sharma, 2014).

O mapeamento de valor e o kaizen reduziu os estoques, (Jasti, \& Sharma, 2014).

Identificação e eliminação de desperdícios

(Carmignani, \& Zammori, 2015 p. 994).

Produção puxada. Foco na qualidade (parar a produção para corrigir erros).

Kanban (Gong, \& Janssen, 2015, p. 371; Kumar Br et al., 2015).

Melhorar o fluxo da produção (Olivella, \& Gregorio, 2015).

Melhorar o fluxo de materiais (Olivella, \& Gregorio, 2015).

Custos são reduzidos pela redução dos estoques devido ao aumento da qualidade (Womack, Jones, \& Roos, 1992).

Total (Somatória dos valores da Coluna) Porcentagem do total (continuação)

\begin{tabular}{|c|c|c|c|c|c|c|c|}
\hline 9 & 9 & 9 & 0 & 9 & 0 & 0 & 3 \\
\hline 3 & 0 & 9 & 0 & 9 & 0 & 3 & 3 \\
\hline 0 & 0 & 1 & 0 & 1 & 1 & 9 & 9 \\
\hline 0 & 0 & 0 & 0 & 1 & 1 & 0 & 0 \\
\hline 9 & 9 & 9 & 9 & 9 & 9 & 9 & 3 \\
\hline 0 & 0 & 9 & 9 & 1 & 9 & 9 & 9 \\
\hline 0 & 0 & 0 & 0 & 0 & 0 & 9 & 9 \\
\hline 0 & 0 & 0 & 0 & 0 & 0 & 9 & 9 \\
\hline 0 & 0 & 1 & 0 & 1 & 1 & 9 & 9 \\
\hline
\end{tabular}

\begin{tabular}{|l|l|l|l|l|l|l|l|}
0 & 0 & 9 & 0 & 0 & 1 & 0 & 0 \\
\hline 0 & 0 & 9 & 0 & 0 & 1 & 0 & 0 \\
\hline 1 & 0 & 1 & 1 & 1 & 0 & 0 & 0 \\
\hline 0 & 0 & 9 & 0 & 0 & 1 & 0 & 0 \\
\hline 1 & 3 & 0 & 3 & 1 & 0 & 0 & 0 \\
\hline
\end{tabular}

\begin{tabular}{ll|l|l|l|l|l|l|}
1 & 3 & 9 & 1 & 9 & 0 & 0 & 0
\end{tabular}

\begin{tabular}{|c|c|c|c|c|c|c|c|}
\hline 3 & 3 & 3 & 3 & 3 & 0 & 0 & 0 \\
\hline 3 & 3 & 3 & 3 & 3 & 0 & 0 & 0 \\
\hline 9 & 3 & 3 & 3 & 1 & 0 & 0 & 0 \\
\hline 9 & 3 & 9 & 9 & 3 & 9 & 9 & 9 \\
\hline 9 & 3 & 9 & 9 & 3 & 9 & 9 & 9 \\
\hline 9 & 3 & 9 & 9 & 3 & 9 & 9 & 9 \\
\hline 3 & 3 & 3 & 3 & 3 & 0 & 0 & 0 \\
\hline 96 & 86 & 143 & 88 & 88 & 79 & 111 & 99 \\
\hline $6,58 \%$ & $5,89 \%$ & $9,80 \%$ & $6,03 \%$ & $6,03 \%$ & $5,42 \%$ & $7,61 \%$ & $6,79 \%$ \\
\hline
\end{tabular}

Exacta, 19(2), p. 432-455, abr./jun. 2021 
(conclusão)

\begin{tabular}{|c|c|}
\hline MA & $\begin{array}{l}\text { Possibilitar a customização em massa (Machado, \& Moraes, 2008; Mondragon et al., 2006; Danese } \\
\& \text { Romano, 2004; Hoek, \& Weken, 1998). }\end{array}$ \\
\hline MC & $\begin{array}{l}\text { Permite ter flexibilidade da linha de manufatura, para variação de demanda e de produto (Spath, } \\
\text { \& Baumeister; 2001). }\end{array}$ \\
\hline $\mathrm{MH}$ & $\begin{array}{l}\text { Apresentar correlação positiva com a porcentagem de entrega em just-in-time (Pérez, \& Sánchez, } \\
\text { 2000). }\end{array}$ \\
\hline MG & Redução do lead time (O'gragy, \& Liang, 1998). \\
\hline MT & Reduzir os custos da customização em massa (Arnheiter, \& Harren, 2005). \\
\hline MV & $\begin{array}{l}\text { O consórcio modular ou condomínio reduz os custos de serviços, como de resolução de problemas } \\
\text { na linha de montagem da montadora (Salerno et al., 2008). }\end{array}$ \\
\hline M3 & Exclusividade de fornecimento (proponente). \\
\hline M4 & $\begin{array}{l}\text { Criar contratos de longos períodos com as montadoras, o que diminui a concorrência (Arnheiter, } \\
\text { \& Harren, 2005; Pires, 2002; Salerno, 2001; Collins et al., 1997; Marx et al., 1997). }\end{array}$ \\
\hline
\end{tabular}

\begin{tabular}{c|c|c|c|c|} 
Escala de correlação & $\begin{array}{c}\text { Efeito neutro: } \\
0\end{array}$ & Efeito fraco: 1 & Efeito médio: 3 & Efeito forte: 9
\end{tabular}

Fonte: Elaborada pelos autores a partir das fontes citadas.

Verifica-se que a Redução do lead time é a correlação de maior valor entre as abordagens da modularidade com o Lean Manufacturing, isto porque o uso de produtos modulares reduz o tempo de produção, montagens e aumenta a flexibilidade da produção, facilitando a aplicação do Just in Time que faz parte da produção Lean Manufacturing. Em segundo lugar, vem Exclusividade de fornecimento, isto porque a terceirização de atividades de projetar e montar os módulos só é possível quando ocorrer uma forte parceria entre o cliente e fornecedor, sendo esta parceria um dos elementos de produção do Lean Manufacturing apresentados por Arnheiter e Greenland (2008); Womack, et al. (1992).

\subsubsection{Análise das duas hipóteses do estudo}

A seguir serão apresentados os resultados desta matriz em relação às duas hipóteses levantadas no estudo, para verificar quais das hipóteses foi confirmada.

Conforme o quadro 1, verifica-se na matriz que dos 30 itens analisados da modularidade, 30 tiveram correlação positiva, com valor total dos pesos entre 9,8\% a 0,75\% em relação aos 37 itens do Lean Manufacturing. Os benefícios da modularidade que tiveram maior correlação com o Lean Manufacturing foram: "Redução do lead time" (O'gragy, \& Liang, 1998); "Exclusividade de fornecimento" (proponente); "Criar contratos de longos períodos com as montadoras, o que diminui a concorrência" (Arnheiter, \& Harren, 2005; Pires, 2002; Salerno, 2001; Collins et al., 1997; Marx et al., 1997) e "Possibilitar a customização em massa" (Machado, \& Moraes, 2008; Mondragon et al., 2006; 
Danese, \& Romano, 2004; Hoek, \& Weken, 1998). Ou seja, fortalece a parceria entre as empresas, aumentando a flexibilidade das mesmas, devido a redução do lead time e facilidade de customização, que são elementos importantes para a aplicação do Lean Manufacturing.

Os resultados indicam que o conjunto dos 30 itens da modularidade tem um efeito levemente positivo em relação à produção Lean Manufacturing. Deste modo, a hipótese 1 foi, parcialmente, confirmada: "O uso da modularidade facilita a aplicação do Lean Manufacturing."

Quadro 1 - Resumo dos resultados das correlações.

Estratégia modular (30 itens)

Resultado da Correlação

O uso da modularidade facilita a aplicação do Lean Manufacturing

Neutro

Fonte: Elaborado pelos autores.

\section{Lean Manufacturing}

(37 itens)

30 de 30

0 de 30

\section{Conclusões}

Após análise dos resultados, verificou-se a alta aplicabilidade da modularidade em diferentes setores, tais como robótica, máquinas $\mathrm{CNC}$, móveis de estações de trabalho, construção de casas, estacionamentos metálicos, prótese para deficientes, desenvolvimento de software, bicicleta, eletrodomésticos, produtos de plásticos, brinquedos, aviões e tratores. Estes setores apresentam ambientes de produção diferentes como: fabricação para estoque (MTS: make to stock); Montagem sob encomenda (ATO: assemble to order); Fabricação sob encomenda (MTO: make to order) e Engenharia sob encomenda (ETO: engineering to order). Deste modo, verificou-se o uso da modularidade em todos os ambientes de produção, o que não significa que o seu efeito é positivo. Verificou-se que se destaca a aplicação nos setores que utilizam o Lean Manufacturing. Os resultados da matriz indicam que a modularidade tem um efeito positivo em relação ao Lean Manufacturing.

Constata-se, portanto, que a hipótese 1 foi, parcialmente, confirmada: "O uso da modularidade facilita a aplicação do Lean Manufacturing". Assim, a abordagem modular não dificulta a aplicação do Lean Manufacturing, podendo ser usadas juntas de forma a contribuir com a aplicação.

Uma possível continuidade deste trabalho seria analisar se o impacto do uso da modularidade também é positivo em ambientes produtivos diferentes do Lean Manufacturing como o ambiente de produção para estoque (MTS: make to stock).

\section{Referências}

Acaccia, G. Bruzzone, L., \& Razzoli, R. (2008). A modular robotic system for industrial applications. Assembly Automation, 28(2), 151-162. doi: https://doi.org/10.1108/01445150810863734. 
Arnheiter, E.D., \& Harren, H. (2005). A typology to unleash the potential of modularity. Journal of Manufacturing Technology Management, 16(7), 699- 711. doi: https://doi.org/10.1108/17410380510619923.

Arnheiter, E. D., \& Harren, H. (2006). Quality management in a modular world. The TQM Magazine, 18(1), 87-96. doi: https://doi.org/10.1108/09544780610637712.

Arnheiter, E. D., \& Greenland, J. E. (2008). Looking for root cause: a comparative analysis. The TQM Journal. 20(1), 18-30. doi: https://doi.org/10.1108/09544780810842875.

Baldwin, C.Y., \& Clark K.B. (2000). Design rules. Massachusetts: MIT Press.

Barbosa, G. F., Carvalho, J., \& Filho, E. V. G. (2014). A proper framework for design of aircraft production system based on lean manufacturing principles focusing to automated processes. The International Journal of Advanced Manufacturing Technology, 72(9-12), 1257-1273. doi: https://doi.org/10.1007/s00170-014-5729-3.

Bevilacqua, M., Ciarapica, F. E., De Sanctis, I., Mazzuto G., \& Paciarotti, C. (2015). A changeover time reduction through an integration of lean practices: a case study from pharmaceutical sector. Assembly Automation, 35(1), 22-34. doi: https://doi.org/10.1108/AA-05-2014-035.

Brookfield, J., Liu, R.J., \& MacDuffie, J.P. (2008). Taiwan's bicycle industry A - Team battles Chinese competition with innovation and cooperation. Strategy \& Leadership, 36(1), 14-19. doi: https://doi.org/10.1108/10878570810840643.

Cagatay Iris, \& Ufuk Cebeci. (2014). Analyzing relationship between ERP utilization and lean manufacturing maturity of Turkish SMEs, Journal of Enterprise Information Management, 27(3), 261-277. doi: https://doi.org/10.1108/JEIM-12-2013-0093.

Carmignani, G., \& Zammori, F. (2015). Lean thinking in the luxury-fashion market evidences from an extensive industrial Project. International Journal of Retail \& Distribution Management, 43(10/11), 988-1012. doi: https://doi.org/10.1108/IJRDM-07-2014-0093.

Carnevalli, J. A., Salerno, M. S., \& Miguel, P. A. C. (2010). Proposta de um levantamento de campo sobre a adoção da modularidade em empresas do setor automotivo visando investigar aspectos sobre a sua aplicação. (Relatório científico do pós-doutorado para a FAPESP) São Paulo, SP, Poli, USP.

Carvalho, M. M., Paladini, E. P., Bouer, G., Ferreira, J.J.D.A., Miguel, P. A. C., Samohyl, R. W., \& Rotondaro, R.G. (2012). Gestão da qualidade: teoria e casos. Rio de Janeiro: Elsevier.

Cauchick Miguel, P. A., \& Pires, S. R. I. (2006). A case study on modularity in product development and production within the auto industry. International Journal of Automotive Technology and Management, 6(3), 315-330. doi: https://doi.org/10.1504/IJATM.2006.012123.

Chaplin, L., Heap J., \& O'rourke, S. T. J. (2016). Reflective practice could "lean lite" be the cost effective solution to applying lean manufacturing in developing economies? International Journal of Productivity and Performance Management, 65(1), 126-136. doi: https://doi.org/10.1108/IJPPM-022015-0034,

Collins, R., Bechler, K., \& Pires, S. R.I. (1997). Outsourcing in the automotive industry: from JIT to Modular Consortia. European Management Journal, 15(5), 498-508. doi: https://doi.org/10.1016/S0263- 2373(97)00030-3. 
Connolly, C. (2008). Prosthetic hands from Touch Bionics. Industrial Robot: An International Journal, 35(4), 290-293. doi: https://doi.org/10.1108/01439910810876364.

Danese, P., \& Romano, P. (2004). Improving inter-functional coordination to face high product variety and frequent modifications. International Journal of Operations \& Production Management, 24(9), 863-885. doi: https://doi.org/10.1108/01443570410552090.

De Mello, A. M., \& Marx, R. (2007). Innovative capacity maintenance by automakers in a product development outsourcing scenario: the case of VW in Brazil. International Journal of Automotive Technology and Management, 7(2/3), 200-215. doi: https://doi.org/10.1504/IJATM.2007.014975.

Doran, D. (2002). Manufacturing for synchronous supply: a case study of Ikeda Hoover Ltd. Integrated Manufacturing Systems, 13(1), 18-24. doi: http://doi.org/10.1108/09576060210411477.

Doran, D. (2005). Supplying on a modular basis: an examination of strategic issues. International Journal of Physical Distribution \& Logistics Management, 35(9), 654-663. doi: http://doi.org/10.1108/09600030510632023.

Fong, A.C.M., \& Hui S.C. (2006). A virtual electronic trading system for business-to-business ecommerce. Kybernetes. 35(6), 865-879. doi: https://doi.org/10.1108/03684920610662674.

Forno, A. J. D., Forcellini, F.A., Kipper, L.M., \& Pereira, F.A. (2016). Method for evaluation via benchmarking of the lean product development process. Benchmarking: An International Journal, 23(4), 792-816. doi: https://doi.org/10.1108/BIJ-12-2013-0114.

Fredriksson, P. (2006a). Operations and logistics issues in modular assembly processes: cases from the automotive sector. Journal of Manufacturing Technology Management, 17(2), 168-186. doi: https://doi.org/10.1108/17410380610642250.

Fredriksson, P. (2006b). Cooperation and conflict in modular production and supplier parks: the case of Volvo Cars' modular assembly system. International Journal of Automotive Technology and Management, 6(3), 298-314. doi: https://doi.org/10.1504/IJATM.2006.012122.

Frigant, V., \& Lung, Y. (2002) Geografical proximity and supplying relationships in modular production. International Journal of Urban and Regional Research, 26(4), 742-755. doi:

http://doi.org/10.1111/1468- 2427.00415.

Gabrieli, L., Cortimiglia, M., \& Ribeiro, J.L. (2007). Modelagem e avaliação de um sistema modular para gerenciamento de informação na Web. Ciência da Informação. 36(1), 35-53. doi: http://dx.doi.org/10.1590/S0100-19652007000100003.

Gong, Y., \& Janssen, M. (2015). Demystifying the benefits and risks of lean service innovation: a banking case study. Journal of Systems and Information Technology, 17(4), 364-380. doi: https://doi.org/10.1108/JSIT-03-2015-0019.

Gu, P., \& Sosale, S. (1999). Product modularization for life cycle engineering. Robotics and Computer Integrated Manufacturing, 15(5), 387-401. doi: https://doi.org/10.1016/S07365845(99)00049-6.

He, D.W., \& Kusiak, A. (1998). Designing an assembly line for modular products. Computers \& Industrial Engineering, 34(1), 37-52. 
Hoek, R.I.V., \& Weken, H.A.M. (1998). The impact of modular production on the dynamics of supply chains. The International Journal of Logistics Management, 9(2), 35-50. doi: https://doi.org/10.1108/09574099810805825.

Holmes, J. (2004). Re-scaling collective bargaining: union responses to restructuring in the North American auto industry. Geoforum, 35(1), 9-21. doi: https://doi.org/10.1016/j.geoforum.2003.06.001.

Jasti, N. V. K., \& Sharma, A. (2014). Lean manufacturing implementation using value stream mapping as a tool. International Journal of Lean Six Sigma. 5(1), 89-116. doi: https://doi.org/10.1108/IJLSS04-2012-0002.

Kumar Br, R., Sharma, M.K., \& Agarwal, A. (2015). An experimental investigation of lean management in aviation. Journal of Manufacturing Technology Management. 26(2), 231-260. doi: https://doi.org/10.1108/JMTM-12-2013-0174.

Lau, A. K. W., \& Yam, R. C. M. (2005). A case study of product modularization on supply chain design and coordination in Hong Kong and China. Journal of Manufacturing Technology Management, 16(4), 432-446. doi: https://doi.org/10.1108/17410380510594516.

Lau, A. K. W., Yam, R.C.M., \& Tang, E.P.Y. (2007). Supply chain product co-development, product modularity and product performance: Empirical evidence from Hong Kong manufacturers. Industrial Management \& Data Systems, 107(7), 1036-1065. doi: https://doi.org/10.1108/02635570710816739.

Lee, W. B., Lau, H., \& Tam, S. (2001). A fuzzy analytic hierarchy process approach in modular product design. Expert Systems, 18(1), 32-42, 2001. doi: https://doi.org/10.1111/1468-0394.00153.

Lohse, N., Ratchev, S., \& Valtchanov, G. (2004). Towards Web-enabled design of modular assembly systems. Assembly Automation, 24(3), 270-279. doi: https://doi.org/10.1108/01445150410549764.

Lopes, R. B., Freitas, F., \& Sousa, I. (2015). Application of lean manufacturing tools in the food and beverage industries. Journal of Technology Management \& Innovation, 10(1), 120-130. doi: http://dx.doi.org/10.4067/S0718-27242015000300013.

Ma, J., \& Kremer, G. E. O. (2016). A systematic literature review of modular product design (MPD) from the perspective of sustainability. The International Journal of Advanced Manufacturing Technology. 86(5-8), 1509-1539.

Machado, A. G. C, \& Moraes, W. F. G. (2008) Estratégias de customização em massa implementadas por empresas brasileiras. Produção, 18(1), 170-183. doi: http://dx.doi.org/10.1590/S010365132008000100013.

Marconi, M. D. A., \& Lakatos, E. M. (2007). Técnicas de pesquisa: planejamento e execução de pesquisas, amostragens e técnicas de pesquisas, elaboração, análise e interpretação de dados. (6a. ed.). São Paulo: Atlas.

Martins, P. G., \& Laugeni, F. P. (2010). Administração da produção. São Paulo: Saraiva.

Marx, R., Zilbovicius, M., \& Salerno, M. S. (1997). The modular consortium in a new VW truck plant in Brazil: new forms of assembler and supplier relationship. Integrated Manufacturing Systems, 8(5), 292-298. doi: https://doi.org/10.1108/09576069710179742. 
Moeckel, R., Jaquier, C., Drapel, K., Dittrich, E., Upegui, A., \& ljspeert, A.J. (2006). Exploring adaptive locomotion with YaMoR, a novel autonomous modular robot with bluetooth interface. Industrial Robot: An International Journal, 33(4), 285-290. doi: https://doi.org/10.1108/01439910610667908.

Mondragon, C. E. C., Mondragon, A.E.C, \& Miller, R. (2006). Modularity, open architecture and innovation: an automotive perspective. International Journal of Automotive Technology and Management, 6(3), 346 - 363. doi: https://doi.org/10.1504/IJATM.2006.012125.

Moore, J. F. (1999). Civil integrated modular avionics - a longer-term view. Aircraft Engineering and Aerospace Technology: An International Journal, 71(6), 550-557. doi: https://doi.org/10.1108/00022669910303702.

Morgan, J. M., \& Liker, J. K. (2006). The TOYOTA product development system: integrating people, process and technology, New York: Productivity Press apud Mund, K., Pieterse, K., \& Cameron, S. (2015). Lean product engineering in the South African automotive industry. Journal of Manufacturing Technology Management, 26(5), 703-724. doi: https://doi.org/10.1108/JMTM-052013-0062.

Morris, D., \& Donnelly, T. (2006). Are there market limits to modularisation? International Journal of Automotive Technology and Management, 6(3), 262- 275. doi: http://doi.org/10.1504/IJATM.2006.012120.

Mund, K., Pieterse, K., \& Cameron, S. (2015). Lean product engineering in the South African automotive industry. Journal of Manufacturing Technology Management, 26(5), 703-724. doi: https://doi.org/10.1108/JMTM-05-2013-0062.

Nahmens, I., \& Ikuma L. H. (2012). Effects of lean construction on sustainability of modular homebuilding. Journal of Architectural Engineering. 18(2), 155-163.

Nepal, B., Monplaisir, L., \& Famuyiwa, O. (2012). Matching product architecture with supply chain design. European Journal of Operational Research. 216(2), 312-325. doi: https://doi.org/10.1016/j.ejor.2011.07.041.

O'gragy, P., \& Liang W. Y. (1998). An object oriented approach to design with modules. Computer Integrated Manufacturing Systems, 11(4), 267-283. doi: https://doi.org/10.1016/S09515240(98)00024-X.

Olivella, J., \& Gregorio, R. (2015). A case study of an integrated manufacturing performance measurement and meeting system. Journal of Manufacturing Technology Management, 26(4), 515535. doi: https://doi.org/10.1108/JMTM-09-2012-0089.

Orsato, R. J., \& Wells, P. (2007). U-turn: the rise and demise of the automobile industry. Journal of Cleaner Production, 15(11-12), 994-1006. doi: https://doi.org/10.1016/j.jclepro.2006.05.019.

Ohfuji, T., Ono, M, \& Akao, Y. (1997). Métodos de desdobramento da qualidade (Vol. 2). Belo Horizonte: Fundação Christiano Ottoni.

Pandremenos, J., Paralikas, J., \& Chryssolouris, G. (2009). Modularity concepts for the automotive industry: A critical review. CIRP Journal of Manufacturing Science and Technology, 1(3), 148-152. doi: https://doi.org/10.1016/j.cirpj.2008.09.012. 
Pérez, M. P., \& Sánchez, A. M. (2000). Lean production and supplier relations: a survey of practices in the Aragonese automotive industry. Technovation, 20(12), 665-676. doi: https://doi.org/10.1016/S0166-4972(00)00011-0.

Pires, S. R. I. (1998). Managerial implications of the modular consortium model in a Brazilian automotive plant. International Journal of Operations \& Production Management, 18(3), 221-232. doi: https://doi.org/10.1108/01443579810368290.

Pires, S. R. I. (2002). New productive systems in the automotive industry: the current situation of three innovative plants Brazil. Internal Journal of Automotive Technology and Management, 2(1), 46-62. doi: https://doi.org/10.1504/IJATM.2002.000056.

Pires, S. R. I., \& Sacomano, M., Neto. (2010). Características estruturais, relacionais e gerenciais na cadeia de suprimentos de um condomínio industrial na indústria automobilística. Produção, 20(2), 172-185. doi: https://doi.org/10.1590/\$0103-65132010005000032.

Ranky, G. N. (2007). Mobile robot sensor and networking design to enable cooperative behavior. Industrial Robot: An International Journal, 34(1), 21-25. doi: https://doi.org/10.1108/01439910710718405.

Rezende, E. M., \& Gouveira, A. M. C. (2006). Sistemas de estacionamento vertical modulado em estrutura metálica. REM: Revista Escola de Minas, 59(3), 279-284. doi:

http://dx.doi.org/10.1590/\$0370-44672006000300005.

Salerno, M. S. (2001). The characteristics and the role of modularity in the automotive business. International Journal of Automotive Technology and Management, 1(1), 92-107. doi: https://doi.org/10.1504/IJATM.2001.000029.

Salerno, M. S., Camargo, O.S., \& Lemos, M.B. (2008). Modularity ten years after: an evaluation of the Brazilian experience. International Journal of Automotive Technology and Management, 8(4), 373381. doi: https://doi.org/10.1504/IJATM.2008.020309.

Sanchez, R., \& Collins, R. P. (2001). Competing - and learning - in modular markets. Long Range Planning, 34(6), 645-667. doi: https://doi.org/10.1016/S0024-6301(01)00099-1.

Shibata, T., Yano, M., \& Kodama, F. (2008). Empirical analysis of evolution of product architecture Fanuc numerical controllers from 1962 to 1997. Research Policy, 34(1), 13-31. doi: https://doi.org/10.1016/j.respol.2004.09.011.

Shuenemann, M., Grosser, V., Leutenbauer, R., Bauer, G., Schaefer, W., \& Reichl, H. (1999). A highly flexible design and production framework for modularized microelectromechanical systems. Sensors and Actuators - A, physical, 73(1-2), 153-168. doi: https://doi.org/10.1109/MEMSYS.1998.659825.

Spath, D., \& Baumeister M. (2001). Synchronisation of material flow and assembly in hybrid and modular systems. Assembly Automation, 21(2), 152-157. doi: https://doi.org/10.1108/01445150110388504.

Veloso, F., \& Fixson, S. (2001). Make-Buy decisions in the auto industry: new perspectives on the role of the supplier as an innovator. Technological Forecasting and Social Change, 67(2-3), 239-257. https://doi.org/10.1016/S0040-1625(00)00092-5. 
Virk, G. S., Muscato, G., Semerano, A., Armanda, M., \& Warren, H.A. (2004). The CLAWAR project on mobile robotics. Industrial Robot: An International Journal, 31(2), 130-138. doi: https://doi.org/10.1108/01439910410522801.

Voordijk, H., Meijboom, B., \& Haan, J. D. (2006). Modularity in supply chains: a multiple case study in the construction industry. International Journal of Operations \& Production Management. 26(6), 600-618. doi: https://doi.org/10.1108/01443570610666966.

Womack, J. P., Jones, D. T., \& Roos.D. (1992). A máquina que mudou o mundo, (5a ed.). Rio de Janeiro: Editora Campus.

Yigit, A., Ulsoy, A.G., \& Allahverdi, A. (2002). Optimizing modular product design for reconfigurable manufacturing. Journal of Intelligent Manufacturing, 13(4), 309-316.

Zhang, G.W., Zhang, S.C., \& Xu, Y.S. (2002). Research on flexible transfer line schematic design using hierarchical process planning. Journal of Materials Processing Technology, 129(1-3), 629-633. doi: https://doi.org/10.1016/S0924-0136(02)00670-2. 